\title{
PD-L1 Inhibitor INCB086550
}

National Cancer Institute

\section{Source}

National Cancer Institute. PD-L1 Inhibitor INCB086550. NCI Thesaurus. Code C158532.

An orally available, small molecule inhibitor of the immunosuppressive ligand, programmed cell death-1 ligand 1 (PD-L1; cluster of differentiation 274; CD274) with potential immune checkpoint inhibitory and antineoplastic activities. Upon administration, PD-L1 inhibitor INCB086550 specifically targets PD-L1 expressed on tumor cells preventing the binding and subsequent activation of its receptor, prog rammed cell death 1 (PD-1; PDCD1; CD279; prog rammed death-1). This reverses T-cell inactivation caused by PD-L1/PD-1 signaling, increases T-cell expansion and enhances the cytotoxic Tlymphocyte (CTL)-mediated anti-tumor immune response against PD-L1-expressing tumor cells. PD-L1, a transmembrane protein expressed on activated T-cells, is overexpressed in some cancer types and plays a significant role in immune evasion by tumor cells. 